\title{
Femtosecond Laser Induced Vortex Anisotropy
}

\author{
Martynas Beresna and Peter G. Kazansky \\ Optoelectronics Research Centre, University of Southampton, SO17 1BJ, United Kingdom \\ pgk@orc.soton.ac.uk
}

\begin{abstract}
Anisotropic bubble chain structures are produced by ultrashort pulse laser irradiation in silica glass. Vortex anisotropy is observed in the irradiated volume. (C2010 Optical Society of America

OCIS codes: (140.3390) Laser materials processing; (260.7120) Ultrafast phenomena
\end{abstract}

Unique properties of the femtosecond laser direct writing offers the potential for realizing photonic devices in a bulk of transparent materials. Depending on the laser intensity and sample material different features with either positive or negative index change or voids can be fabricated thus targeting numerous applications such as integrated optics, data storage, and diffractive optical elements. It has been shown that structures written in silica glass above a certain intensity threshold with linearly polarized laser beam exhibit form birefringence [1] related to periodic nanostructures self-assembled within the irradiated volume [2]. Recently, nano-sized chiral structures formed with circular polarization have been reported [3]. Anisotropy in glass can be also produced by stress [4] or reflection from an interface. Investigation of the anisotropy origin is important for elucidating the mechanisms of light-matter interaction and device fabrication. Here we report observation of unusual vortex anisotropy in silica glass after femtosecond laser irradiation.

In our experiment the radiation from a regeneratively amplified mode-locked $\mathrm{Yb}: \mathrm{KGW}$ laser (270 fs pulse duration, $200 \mathrm{kHz}$ repetition rate), operating at a wavelength $1030 \mathrm{~nm}$, was focused via $\times 100(\mathrm{NA}=0.7)$ microscope objective into silica sample, which was mounted on a 3-axis motorized translation stage. Circular polarization was chosen to avoid form birefringence originating from nanogratings. The exposure time was in the range from $4 \mu \mathrm{s}$ to $20 \mathrm{~s}$ and the pulse energy was $1.6 \mu \mathrm{J}$. A series of dots was written about $60 \mu \mathrm{m}$ below the surface, while the sample was translated parallel to the polished edge and perpendicular to the laser beam.

After irradiation the sample was inspected with optical microscope and quantitative birefringence measurement system Abrio (CRi Inc.). The structures were analyzed in parallel (Fig. 1) and transverse (Fig. 2) planes of light propagation. In the structure's tail, relatively strong birefringence was induced by the material compaction (Fig. 1c), which is typical behaviour for silica glass when small energy dose is deposited; this well coheres with the assumption that the tail is formed by the very beginning of incident laser pulse. In the central part, however, where higher intensity is reached, expansion occurs leading to radial stress distribution around the structure. Inside the irradiated volume, material is evenly distributed: no stress related birefringence was observed at all examined writing conditions.
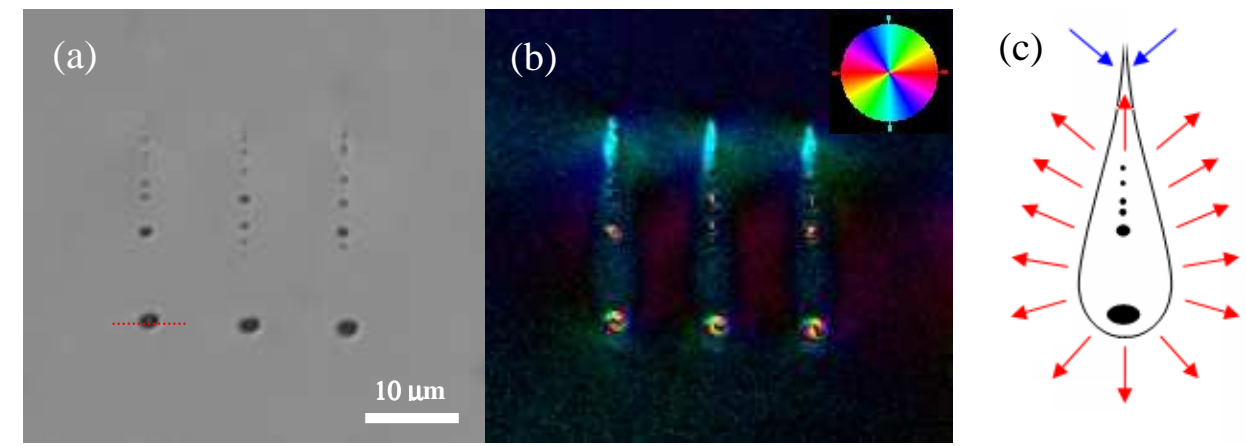

Fig. 1. (a) Optical image of the structures formed by femtosecond laser in silica glass (exposure time $0.01 \mathrm{~s}$ ). Red dashed line indicates plane where vortex anisotropy is observed. (b) Corresponding Abrio image in pseudo color showing direction of the slow axis (see inset). (c) Stress direction deduced from the Abrio image: red arrows indicate stress due to expansion, blue arrows indicate compaction.

Formation of bubble chain along light propagation direction was also observed, which origin is still under discussion [5-6]. In structures written with only several pulses, bubbles were evenly distributed along the central axis. At longer exposure (50 and more pulses per dot) separation between bubbles started to increase. After $10^{4}$ pulses one big bubble was formed at the head of induced structure. Probably, all bubbles are formed during the first 
pulse. After subsequent pulses most of them disappear during surrounding material redistribution. The only bubble at the head continues to grow with exposure time.

In the perpendicular plane peculiar vortex-like features were observed at the head of structure, where the large bubble is formed (Fig. 2).

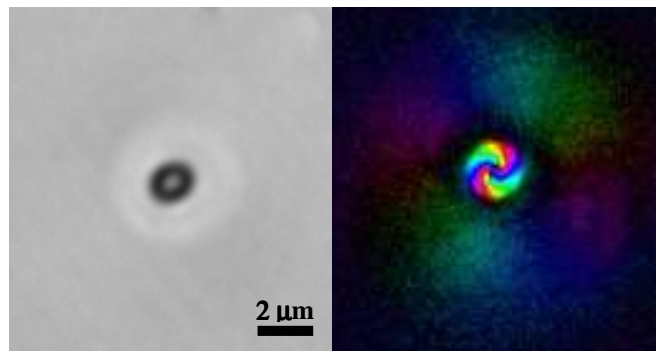

Fig. 2. Optical and Abrio images (exposure time $1 \mathrm{~ms}$ ). The large color ring is formed by the material expansion induced radial stress.

This chirality could be only observed with Abrio system. The handedness of the vortex could not be reversed by changing circular polarization direction. Similar features were also observed in structures produced by linearly polarized light, however they were strongly obscure by form birefringence. Identical chiral features were found in the structures produced at the same experimental conditions with Ti:Sapphire laser $\left(\lambda=800 \mathrm{~nm}\right.$, $\left.\tau_{\text {pulse }}=150 \mathrm{fs}\right)$. Quantitative birefringence measurements are based on characterization of transmitted light ellipticity. It is know that polarization state can be changed due to birefringence or reflection from an interface. Sharp vortex boundaries and its development with diffraction prompt that in this case the anisotropy is produced by the interface possessing chirality.

Arrays of such vortex structures could be used as polarization state converters for optical trapping and manipulation (Fig. 3).

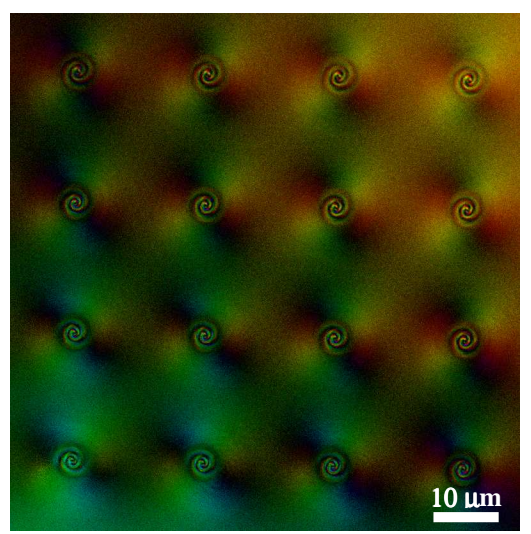

Fig. 3. Array of anisotropic vortices

The puzzle of discovered vortex anisotropy formation is under investigation.

\section{References}

[1] E. Bricchi, B. G. Klappauf, and P. G. Kazansky, "Form birefringence and negative index change created by femtosecond direct writing in transparent materials," Opt. Lett. 29, 119-121 (2004).

[2] Y. Shimotsuma, P. G. Kazansky, J. Qiu, and K. Hirao. "Self-organized nanogratings in glass irradiated by ultrashort light pulses," Phys. Rev. Lett. 24, 2474051-2474054 (2003).

[3] R. S. Taylor, E. Simova, and C. Hnatovsky, "Creation of chiral structures inside fused silica glass," Opt. Lett. 33, 1312-1314 (2008).

[4] B. Poumellec, L. Sudrie, M. Franco, B. Prade, and A. Mysyrowicz, "Femtosecond laser irradiation stress induced in pure silica," Opt. Express 11, 1070-1079 (2003).

[5] E. Toratani, M. Kamata, and M. Obara, "Self-fabrication of void array in fused silica by femtosecond laser processing," Appl. Phys. Lett. 87, 171103 (2005).

[6] S. Kanehira, J. Si, J. Qiu, K. Fujita, and K. Hirao, "Periodic nanovoid structures via femtosecond laser irradiation,” Nano Lett. 5, 1591-1595 (2005). 